\title{
Classification of Boundary Equilibrium Bifurcations in planar Filippov systems
}

Glendinning, Paul

2015

MIMS EPrint: 2015.50

Manchester Institute for Mathematical Sciences

School of Mathematics

The University of Manchester

\footnotetext{
Reports available from: http://eprints.maths.manchester.ac.uk/

And by contacting: The MIMS Secretary

School of Mathematics

The University of Manchester

Manchester, M13 9PL, UK
} 


\section{Classification of Boundary Equilibrium Bifurcations in planar Filippov systems}

Paul Glendinning ${ }^{1, a)}$

School of Mathematics, University of Manchester, Manchester M13 9PL, U.K.

(Dated: June 2015)

If a family of piecewise smooth systems depending on a real parameter is defined on two different regions of the plane separated by a switching surface then a boundary equilibrium bifurcation occurs if a stationary point of one of the systems intersects the switching surface at a critical value of the parameter. We derive the leading order terms of a normal form for boundary equilibrium bifurcations of planar systems. This makes it straightforward to derive a complete classification of the bifurcations that can occur. We are thus able to confirm classic results of Filippov ${ }^{5}$ using different and more transparent methods, and explain why the 'missing' cases of Hogan et $a l^{7}$ are the only cases omitted in more recent work.

The dynamics of a piecewise smooth system are determined by different equations according to which regions of phase space the solutions pass through. In each region the evolution is defined by a smooth dynamical system, but the defining system changes as solutions cross boundaries between regions ('switching surfaces'). Piecewise smooth systems arise naturally in mechanics, biology, control theory and electronics. Even so, the conceptual framework for understanding changes in dynamics as parameters are varied, i.e. bifurcation theory, is still being developed. The simple cases for planar systems in which an equilibrium of one system intersects a switching surface at a critical parameter value were described by Filippov in his seminal book ${ }^{5}$. More recent accounts have often been incomplete, and Hogan et al identify two 'missing' cases ${ }^{7}$. In this paper a lowest order normal form is derived that make the systematic classification of cases much easier to analyze and demonstrates that Filippov's list is indeed complete. An important codimension two case that arises repeatedly in the analysis is also described.

\section{INTRODUCTION}

Suppose that a surface divides phase space (the Euclidean plane in the analysis below) into two regions, and that within each region a smooth differential equation describes the evolution of trajectories in that region. The standard theory of differential equations applies to a trajectory until it strikes the boundary between the regions. Here one of three conditions holds. If the vector fields of both differential equations are transverse to the surface with normal components in the same direction then the trajectory crosses the surface continuously, but with a possible jump in the tangent. If the vector fields

a) Electronic mail: p.a.glendinning@manchester.ac.uk of both differential equations are transverse to the surface with normal components in the opposite directions then the trajectory 'sticks' to the surface and a new rule (or rules) for its evolution need to be determined. Finally, the trajectory may be tangential to the surface at the intersection; this is called grazing and may result in non-unique solutions either following the solution back into the region from which it came, or into the surface as in the previous case, or through the surface into the other region, depending on a more detailed description of the tangency. One possible strategy to treat the 'sticking' solutions on the surface is to assume that a linear combination of the two vector fields is taken so that the transverse component of this combination is zero. This defines the sliding, or Filippov, flow.

At first sight this may look outlandish, but many problems in control theory (where interventions are switched off and on in different parts of phase space), mechanics (where impacts lead to jumps in phase space), electronic circuits and biological modelling all use piecewise smooth systems of the type described above or their generalizations ${ }^{1}$. Filippov ${ }^{5}$ wrote a seminal description of the theory of these systems, although many results are stated without complete explication. As a result, many of the phenomena described by Filippov ${ }^{5}$ have been rediscovered independently in the past few years, often in partial form.

The codimension one bifurcations of planar piecewise smooth systems involving a stationary point on the switching manifold is one such example. Filippov ${ }^{5}$ states that there are eight codimension one flows of this type and sketches the vector fields without detailed derivation of the cases. He goes on (Filippov ${ }^{5}$ pp. 246-250) to give a detailed analysis of the different cases for general systems. The approach below is designed to be much clearer about how the different cases arise by using a normal form (or more precisely the lowest order terms of a normal form) on which the analysis is more transparent. Kuznetsov et $a l^{8}$ give an excellent description of some of the bifurcations that can occur, but their list is incomplete. Guardia et $a l^{6}$ skip past the simple cases, referring to Kuznetsov et $a l^{8}$ for details, and concentrate on unfolding singularities in more than one parameter. Codimension two bifurcations are also the focus of other 
studies $^{2,4}$. Recently Hogan et $a l^{7}$ have made a careful study of the results of Filippov and produce a complete list of the bifurcations, including two that had been overlooked by many authors. They also provide an example in which all the known bifurcations are realized. However, there is still no systematic development of the theory of these codimension one bifurcations of boundary equilibria that makes it clear why there are eight nonhyperbolic cases, nor why Filippov's list of bifurcations is exhaustive.

This paper presents a new framework for the systematic analysis of these bifurcations. An affine change of variable and a change of the direction of time in some cases is used to simplify the linear part of the vector field on one side of the switching manifold in such a way that the Filippov sliding flow is also preserved (this is the extra subtlety that is needed to ensure that the analysis is correct). These simplified equations, essentially the lowest order truncations of a normal form for the problem, are then analyzed, and there are few enough cases that this can be done without a huge effort. This yields the bifurcations of Filippov. The change of variable is reminiscent of the approach taken by Nusse and Yorke in their derivation of the border collision normal form ${ }^{9}$. The linear part we use is also essentially that of Carvalho and Tonon ${ }^{3}$, with the addition of a parameter to unfold the bifurcations.

The remainder of the paper is organized as follows. In the next section the formal description of the systems discussed is given, together with the definition of the Filippov flow. The general conditions for a codimension one boundary equilibrium bifurcation are also given. In section III a series of coordinate changes is used to rewrite a general system locally in a particularly simple form which has hyperbolic behaviour away from the bifurcation point making it possible to concentrate on the local truncation to determine the dynamics near the bifurcation. This local truncation is

$$
\left(\begin{array}{c}
\dot{x} \\
\dot{y}
\end{array}\right)= \begin{cases}L_{k}\left(\begin{array}{c}
x \\
y-\mu
\end{array}\right) & \text { if } y>0 \\
\left(\begin{array}{c}
c \\
1
\end{array}\right) & \text { if } y<0\end{cases}
$$

with $k \in\{0,1\}$ and

$$
L_{0}=\left(\begin{array}{ll}
a & b \\
b & a
\end{array}\right) \quad \text { and } \quad L_{1}=\left(\begin{array}{cc}
a & -b \\
b & a
\end{array}\right)
$$

Here the signs of the constants $a$ and $b$ (both non-zero), and $c$ can be chosen in various ways (in particular not all cases need to be considered and, for example, without loss of generality $b>0$ ) and $\mu$ is the parameter. The choice of $L_{0}$ gives real eigenvectors $a \pm b$ and the choice of $L_{1}$ gives complex eigenvalues $a \pm i b$. In the following sections the different possibilities arising from (1) are described, leading to the identification of the codimension one bifurcations which are summarized in section X. Sec- tion XI describes a codimension two case that recurs as a boundary condition in the analysis of the earlier sections.

\section{BOUNDARY EQUILIBRIUM BIFURCATIONS}

In general the switching manifold is a function $H(x, y, \mu)=0$ and we assume that at the bifurcation value of the parameter an equilibrium of one of the defining vector fields lies on the switching manifold. This is itself a codimension one condition and so to analyze the codimension one bifurcations of such points all the other properties of the flow must be generic. Provided the defining functions are sufficiently smooth a change of variable can be made so that the bifurcation occurs at the origin in phase space when $\mu=0$, and that the switching manifold is $y=0$. Thus after these changes of variable the system is

$$
\left(\begin{array}{l}
\dot{x} \\
\dot{y}
\end{array}\right)= \begin{cases}f^{+}(x, y, \mu) & \text { if } y>0 \\
f^{-}(x, y, \mu) & \text { if } y<0\end{cases}
$$

where $f^{ \pm}: \mathbb{R}^{2} \times \mathbb{R} \rightarrow \mathbb{R}^{2}$ are smooth functions (in particular both can be extended to the whole plane) and

$$
f^{+}(0,0,0)=(0,0)^{T}
$$

where a simple shift in the $x$-direction has been used so that the intersection of the stationary point with the switching manifold $y=0$ at $\mu=0$ is at the origin. Expanding about the origin

$$
\begin{aligned}
f^{+}(x, y, \mu)=\left(\begin{array}{c}
V_{1} \\
V_{2}
\end{array}\right) \mu & +\left(\begin{array}{cc}
A & B \\
C & D
\end{array}\right)\left(\begin{array}{c}
x \\
y
\end{array}\right) \\
& +O\left(|(x, y, \mu)|^{2}\right),
\end{aligned}
$$

and

$$
f^{-}(x, y, \mu)=\left(\begin{array}{c}
U_{1} \\
U_{2}
\end{array}\right)+O(|(x, y, \mu)|)
$$

For generic systems the constant $U_{2}$ is non-zero, the ma$\operatorname{trix}\left(\begin{array}{cc}A & B \\ C & D\end{array}\right)$ has distinct eigenvalues with non-zero real parts and

$$
C \neq 0 \text {. }
$$

The first of these conditions ensures that the lower flow is not tangential to the $x$-axis locally, the second is a standard genericity assumption, and the third, (7), implies that the flow in the $y$-direction does not decouple from the $x$-variable to lowest order; geometrically it implies that neither eigenvector of the linear part of the equations is parallel to the $x$-axis. The direction of time can be chosen so that

$$
U_{2}>0
$$

and so there is a neighbourhood of the origin for which all trajectories of the lower dynamical system point upwards 
across $y=0$ and so the section of the switching manifold $y=0$ in this neighbourhood on which sliding motion is defined (if it exists) is that for which the $y$-component of $f^{+}$points downwards (i.e. $\left.f^{+}(x, 0, \mu) \cdot(0,1)^{T}<0\right)$.

Write $f^{ \pm}$in component form as $\left(f_{1}^{ \pm}, f_{2}^{ \pm}\right)^{T}$. Suppose that there is a line segment on which $f_{2}^{+}(x, 0, \mu)<0$ for some fixed $\mu$ (and $f_{2}^{-}(x, 0, \mu)>0$ by assumption). The Filippov construction suggests defining the evolution on this stable switching surface by setting

$$
\dot{x}=q f_{1}^{-}(x, 0, \mu)+(1-q) f_{1}^{+}(x, 0, \mu)
$$

where $q$ is chosen so that the net $y$ evolution is zero, i.e.

$$
q f_{2}^{-}(x, 0, \mu)+(1-q) f_{2}^{+}(x, 0, \mu)=0 .
$$

Solving for $q$ and substituting back into (9) gives

$$
\dot{x}=\frac{f_{2}^{-}(x, 0, \mu) f_{1}^{+}(x, 0, \mu)-f_{2}^{+}(x, 0, \mu) f_{1}^{-}(x, 0, \mu)}{f_{2}^{-}(x, 0, \mu)-f_{2}^{+}(x, 0, \mu)}
$$

which is called the Filippov or sliding flow on the switching manifold ${ }^{1,5}$. Assuming that $f_{2}^{+}(x, 0, \mu)<0$, i.e. $V_{2} \mu+C x+O\left(|(x, \mu)|^{2}\right)<0$ then the Filippov flow for (3) is (ignoring the higher order terms in both numerator and denominator)

$$
\dot{x}=\frac{U_{2}\left(A x+V_{1} \mu\right)-\left(V_{2} \mu+C x\right) U_{1}}{U_{2}-\left(V_{2} \mu+C x\right)} .
$$

A stationary point of the sliding flow will be called a pseudo-stationary point of the full system. We will refer to the linear type of a pseudo-stationary point in the obvious way (pseudo-saddle, pseudo-node...).

There are other conventions for defining flows on and through the switching manifold, for example using differential inclusions on convex combinations of the vector field, but here we restrict attention to the standard choice of (11).

\section{COORDINATE TRANSFORMATIONS}

To simplify the form of the map we will use invertible affine coordinate transformations. These need to be chosen so that the positive and negative $y$ half-planes map to themselves, so that the switching surface is preserved, and also such that the Filippov flow on any sliding surfaces are also preserved.

Consider the change of variable

$$
\left(\begin{array}{l}
X \\
Y
\end{array}\right)=\left(\begin{array}{ll}
\alpha & \beta \\
0 & \gamma
\end{array}\right)\left(\begin{array}{l}
x \\
y
\end{array}\right)
$$

with inverse

$$
\left(\begin{array}{l}
x \\
y
\end{array}\right)=\frac{1}{\alpha \gamma}\left(\begin{array}{cc}
\gamma & -\beta \\
0 & \alpha
\end{array}\right)\left(\begin{array}{l}
X \\
Y
\end{array}\right)
$$

The upper and lower half planes are mapped to themselves if

$$
\gamma>0
$$

and a short calculation shows that equation (5) for the vector field in $y>0$, or equivalently $Y>0$, becomes

$$
\left(\begin{array}{c}
\dot{X} \\
\dot{Y}
\end{array}\right)=\left(\begin{array}{c}
\alpha V_{1}+\beta V_{2} \\
\gamma V_{2}
\end{array}\right) \mu+L\left(\begin{array}{c}
X \\
Y
\end{array}\right)+O\left(|(X, Y, \mu)|^{2}\right)
$$

where $L$ is the matrix

$$
\frac{1}{\alpha \gamma}\left(\begin{array}{cc}
\gamma(\alpha A+\beta C) & \alpha \beta(D-A)+\alpha^{2} B-\beta^{2} C \\
\gamma C & \gamma(\alpha D-\beta C)
\end{array}\right) .
$$

This matrix will have equal entries on the diagonal as required by (1) if $\alpha(D-A)=2 \beta C$. By assumption (7) $C \neq 0$ and so

$$
\beta=\frac{\alpha(D-A)}{2 C} .
$$

The off-diagonal terms of the normal form (1) in $y>0$ have equal modulus and if the eigenvalues of the linear part are real then they are equal. In the case of real distinct eigenvalues

$$
\Delta=(A+D)^{2}-4(A D-B C)>0
$$

and equality of the off-diagonal coefficients of (17) implies

$$
\gamma=\frac{\alpha^{2} \Delta}{4 C^{2}}, \quad(\Delta>0)
$$

which is greater than zero as required. So $\gamma$ determines $\alpha$ and $\beta$ by (20) and (18) up to a sign which is fixed by choosing $\alpha>0$ so that the $x$-axis is mapped to itself preserving orientation. If the eigenvalues are non-zero and purely imaginary then $\Delta<0$ and the off-diagonal elements have opposite signs provided

$$
\gamma=-\frac{\alpha^{2} \Delta}{4 C^{2}}, \quad(\Delta<0)
$$

which is again positive and once again $\gamma$ determines $\alpha$ and $\beta$ up to a sign. Note that $L$ does not depend on the signs chosen.

With these choices,

$$
L=\left(\begin{array}{cc}
\frac{1}{2}(A+D) & \pm C / \gamma \\
C / \gamma & \frac{1}{2}(A+D)
\end{array}\right)
$$

and the new variables evolve under the vector field $F^{+}$ defined by (16), whilst in these new coordinates the lower vector field becomes $F^{-}$which is

$$
\left(\begin{array}{c}
\dot{X} \\
\dot{Y}
\end{array}\right)=\left(\begin{array}{c}
\alpha U_{1}+\beta U_{2} \\
\gamma U_{2}
\end{array}\right)+O(|(x, y, \mu)|) .
$$

One degree of freedom remains in the choice of $\gamma$, reversing time has already been used to fix the sign of $U_{2}$, see (8), and it will also be possible to shift and/or reverse the sign of $X$ if needed.

First consider the sign of the components transverse to $y=0$. By definition $Y=\gamma y$ and so $\dot{Y}=\gamma \dot{y}$ and so the 
time derivative of the transformed second coordinate has the same sign as the original coordinate. More precisely $\dot{\mathbf{x}}=f^{ \pm}(\mathbf{x})$ with the choice of $\operatorname{sign}$ on $f^{ \pm}$determined by the sign of $y$ and hence $Y$, and $\dot{\mathbf{X}}=F^{ \pm}(\mathbf{X})=S f^{ \pm}(\mathbf{x})$ where $\mathbf{x}=S^{-1} \mathbf{X}$. Taking the second coordinate shows $\left.\dot{Y}\right|_{(X, Y)}=\gamma f_{2}^{ \pm}(\mathbf{x})$. In other words the $Y$ derivative in the new coordinates is $\gamma$ times the $y$ derivative at the preimage of that point in the old coordinates and so $\gamma>$ 0 ensures that the signs are the same at corresponding points.

On the stable portions of the switching manifold, which are images of each other under the argument of the previous section, the Filippov sliding flows (11) are

$$
\dot{x}=\frac{f_{2}^{-} f_{1}^{+}-f_{2}^{+} f_{1}^{-}}{f_{2}^{-}-f_{2}^{+}}, \quad \dot{X}=\frac{F_{2}^{-} F_{1}^{+}-F_{2}^{+} F_{1}^{-}}{F_{2}^{-}-F_{2}^{+}} .
$$

Now we have already seen that $F_{2}^{ \pm}=\gamma f_{2}^{ \pm}$at the corresponding points, and $F_{1}^{ \pm}=\alpha f_{1}^{ \pm}+\beta f_{2}^{ \pm}$so on the sliding surface

$$
\dot{X}=\frac{\gamma f_{2}^{-}\left(\alpha f_{1}^{+}+\beta f_{2}^{+}\right)-\gamma f_{2}^{+}\left(\alpha f_{1}^{-}+\beta f_{2}^{-}\right)}{\gamma\left(f_{2}^{-}-f_{2}^{+}\right)}
$$

i.e.

$$
\dot{X}=\alpha \frac{f_{2}^{-} f_{1}^{+}-f_{2}^{+} f_{1}^{-}}{f_{2}^{-}-f_{2}^{+}}
$$

where the right hand side is evaluated at $x=X / \alpha$. This is exactly the equation required to preserve the flow on the sliding manifold. To see this suppose that $\dot{x}=g(x)$ is the sliding flow in the original coordinates. Then the coordinate transformation (13) restricted to the sliding manifold $y=0$ is $X=\alpha x$ and hence $\dot{X}=\alpha g(X / \alpha)$ is the conjugate flow, and this is precisely (24). This result is not new and can be found for example in Filippov's book $^{5}$ (Corollary to Theorem 4, p. 105).

By choosing

$$
\gamma=1 / U_{2}
$$

the second component of the lower vector field is set to unity to leading order.

With this choice we are one step away from completing the derivation of the leading order normal form (1). To be clear, in the new coordinates the piecewise smooth system is

$$
\begin{aligned}
F^{+}(X, Y, \mu) & =\left(\begin{array}{c}
\alpha V_{1}+\beta V_{2} \\
V_{2} / U_{2}
\end{array}\right) \mu \\
& +\left(\begin{array}{cc}
\frac{1}{2}(A+D) & \pm U_{2} C \\
U_{2} C & \frac{1}{2}(A+D)
\end{array}\right)\left(\begin{array}{c}
X \\
Y
\end{array}\right) \\
& +O\left(|(X, Y, \mu)|^{2}\right)
\end{aligned}
$$

and

$$
F^{-}(X, Y, \mu)=\left(\begin{array}{c}
\alpha U_{1}+\beta U_{2} \\
1
\end{array}\right)+O(|(X, Y, \mu)|) .
$$

The final freedom remaining is to choose the direction of $X$ so that the sliding manifold is always on the left; this will make comparisons between different cases much easier to understand. Since the lower vector field always has an upwards component, the switching manifold will lie on the side which has $F_{2}^{+}<0$ when $Y=0$ (i.e. on the switching manifold). Thus we need to consider the sign of

$$
\left(V_{2} / U_{2} \mu+U_{2} C X\right)
$$

for large $|X|$ and make sure this is negative. If $U_{2} C>0$ (i.e. $C>0$ ) then write $a=\frac{1}{2}(A+D)$ and $b=U_{2} C>0$ and do not change the orientation of $X$. If $U_{2} C<0$ set $b=-U_{2} C>0$ and reverse the direction of $X$, setting $\tilde{X}=-X$ and then dropping the tilde immediately. The effect of this is that in the new coordinates

$$
\begin{aligned}
F^{+}(X, Y, \mu)= & \left(\begin{array}{c}
\sigma\left(\alpha V_{1}+\beta V_{2}\right) \\
V_{2} / U_{2}
\end{array}\right) \mu \\
& +\left(\begin{array}{c}
a \pm b \\
b
\end{array}\right)\left(\begin{array}{c}
X \\
Y
\end{array}\right) \\
& +O\left(|(X, Y, \mu)|^{2}\right),
\end{aligned}
$$

and

$$
\begin{aligned}
F^{-}(X, Y, \mu)= & \left(\begin{array}{c}
\sigma\left(\alpha U_{1}+\beta U_{2}\right) \\
1
\end{array}\right) \\
& +O(|(X, Y, \mu)|)
\end{aligned}
$$

where $\sigma=+1$ if $C>0$ and $\sigma=-1$ if $C<0$. Finally, a shift in the $X$-direction of order $\mu$ can be used to write the parameter dependence as in (1). To see this simply note that if $Z=X-p \mu$ for some constant $p$ and

$$
\dot{X}=a X+\sigma b Y+r \mu, \quad \dot{Y}=b X+a Y+s \mu
$$

where $\sigma \in\{+1,-1\}$ and $r$ and $s$ are constants then

$$
\dot{Z}=a Z+\sigma b Y+(a p+r) \mu, \quad \dot{Y}=b Z+a Y+(b p+s) \mu
$$

and if $p$ is chosen so that $(b p+s) /(a p+r)=a / b$ then $(b p+s) / a=(a p+r) / b=k$, say and so $a p+r=k b$, $b p+s=k a$ and the equation becomes

$$
\dot{Z}=a Z+\sigma b Y+k b \mu, \quad \dot{Y}=b Z+a Y+k a \mu
$$

or

$$
\left(\begin{array}{c}
\dot{Z} \\
\dot{Y}
\end{array}\right)=\left(\begin{array}{cc}
a & \sigma b \\
b & a
\end{array}\right)\left(\begin{array}{c}
Z \\
Y+k \mu
\end{array}\right)
$$

from which we obtain the truncated normal form by redefining $\mu$ by a factor of $-k$ and relabelling the variables in the obvious way. Note that this order $|\mu|$ shift does not change the form of the leading order terms of the lower vector field.

A great advantage of this formulation is that in the real case the stationary point is at $(0, \mu 0$, the eigenvalues of the linear part are simply $a \pm b$ and the corresponding eigenvectors are the lines $y=\mu \pm x$. This makes the conditions for intersections of these lines with the switching manifold particularly easy to describe: if $\mu>0$ it they are simply the points $( \pm \mu, 0)$. 


\section{FILIPPOV'S EIGHT}

For the remainder of this paper we assume that the coordinate transformation of the previous section has been made so that any generic Filippov system has leading order terms given by (1) with linear parts (2).

Filippov describes the vector fields at a boundary equilibrium bifurcation as a type 4 singularity of planar piecewise smooth systems and identifies eight of these (up to conjugacy). These are reproduced here in a slightly modified form for clarity - the convention in this paper that the sliding manifold is a half-line extending in $x<0$ makes the complete classification more obvious. Essentially the local phase portraits of Figure 1 can be understood from the normal form (1) by considering the different alternatives for the linear type of the singular point at $\mu=0$ of the upper vector field $f^{+}$together with the direction of the Filippov flow on the sliding manifold at the bifurcation point.

With the convention of the previous paragraph there are four types of stationary point (stable and unstable nodes, foci, and saddles - the stability of the focus does not affect the diagram here) and in each of these the direction of the flow on the sliding manifold may either be positive or negative in the $x$-direction. These determine the eight codimension one vector fields of type 4 described by Filippov ${ }^{5}$.

Note that in the figure the lower vector field is drawn as vertical if $c$ may take both positive or negative values (for appropriate values of the other parameters), whilst it is drawn in the direction determined by $c$ in the cases where only one sign of $c$ is possible. This follows what appears to be Filippov's convention, although he does not use it on the saddles. It is a relatively easy exercise to determine the range of parameters in the normal form (1) appropriate for the different cases. These are summarized below in the order that the cases are considered in this paper. By the calculation of (36) made in the section $\mathrm{V}$ if the linear part of the normal form is given by $L_{0}$ in (2), and of (44) in section VIII if the normal form is given by $L_{1}$, the sign of the flow on the sliding surface $y=0$, $x<0$ is the sign of $(a-b c) x$ if $\mu=0$. The different cases reflect the labelling of Figure 1.

- Stable node: $a<0,0<b<|a|$ and $c>\frac{a}{b}$ in case (a) and $c<\frac{a}{b}$ in case (b). Note that since $a<0$ the sign of $c$ in case (b) must be negative.

- Unstable node: $0<b<a$ and $c<\frac{a}{b}$ in case(c); $c>\frac{a}{b}$ in case (d) and the sign of $c$ must be positive in case (d).

- Saddle: There are two possible cases for the saddle, either $0<a<b$ or $0>a>-b$. If $\dot{x}>0$ on the switching surface as in Figure 1e then $c>a / b$ which may be either positive or negative if $0>a>-b$ (and is always positive if $0<a<b)$. Similarly $\dot{x}<0$ on the switching surface as in Figure 1e then $c<a / b$ which
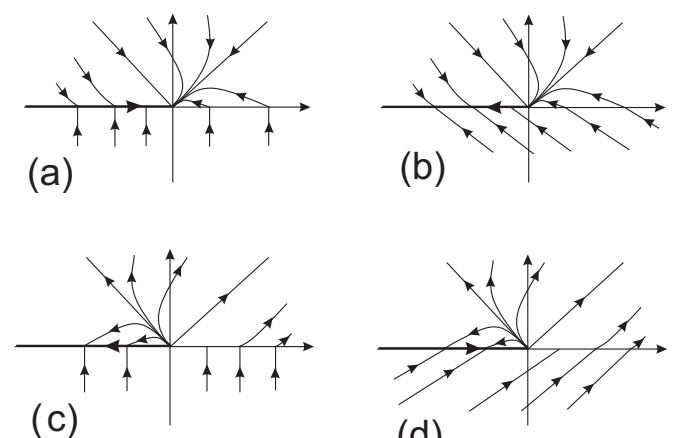

(d)
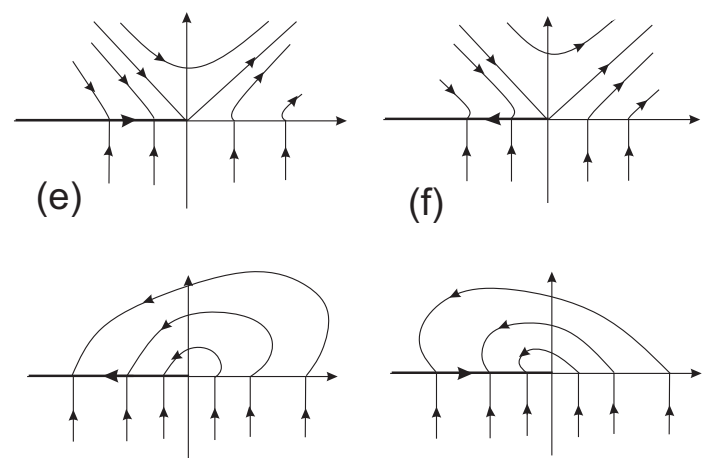

(g)

(h)

FIG. 1. The eight codimension one phase portraits with the convention that vertical lines are used in $y<0$ if both signs of $c$ are possible, otherwise the slope indicates the sign of $c$. The direction of time has been chosen so that the sliding manifold is $y=0$ with $x<0$, so for comparison the cases (a)-(h) correspond to Figures 91, 93, 94, 92, 89, 90, 95, 96 respectively.

may be either positive or negative if $0<a<b$ (and is always negative if $0>a>-b$ ).

- Focus: In this case the linear part is given by $L_{1}$ in (2) and $a<0$ for the stable focus and $a>0$ for the unstable focus. Thus both signs of $c$ are possible in both cases depending on the sign of $a$.

\section{STABLE NODE}

This section will be relatively detailed, but in later sections the results of routine calculations will be stated rather than derived. If the hyperbolic stationary point of $f^{+}$is a stable node ( $\mathrm{SN}$ ) then the eigenvalues $a \pm b$ of the linear part of the symmetric matrix $L_{0}$ version of (1) with (2) must satisfy

$$
a<0, \quad 0<b<|a|
$$

With this choice both eigenvalues of $L_{0}, a \pm b$, are negative and distinct and the eigenvectors are $\left(\begin{array}{c}1 \\ \pm 1\end{array}\right)$. The stable node exists if $\mu>0$. To understand the dynamics it is necessary to understand the flow on the Filippov surface 
and how this varies with $\mu$ and the other parameters. Recall from (1) that to lowest order

$$
\dot{x}=a x+b(y-\mu), \quad \dot{y}=b x+a(y-\mu), \quad \text { if } y>0
$$

and

$$
\dot{x}=c, \quad \dot{y}=1, \quad \text { if } y<0 .
$$

Since $\dot{y}>0$ if $y<0$ the Filippov sliding surface is obtained from the condition $\dot{y}<0$ on $y=0$ from the flow in $y>0$ i.e. $b x-a \mu<0$ and since $b>0$ this means

$$
x<x_{T}=\frac{a}{b} \mu .
$$

It will be useful (to avoid messy equations) to define

$$
s=\frac{a}{b}
$$

and note that $s<-1$ in this section as $a<-b<0$. The Filippov sliding flow of the truncated normal form in $x<s \mu$ is

$$
\dot{x}=\frac{1}{D}((a-b c) x+(a c-b) \mu)
$$

where

$$
D=1-b x+a \mu
$$

and $D>0$ in a neighbourhood of the origin. There is a pseudo-equilibrium (a solution of $\dot{x}=0$ on the sliding manifold $\left.x<x_{T}\right)$ at

$$
x_{P S}=\left(\frac{b-a c}{a-b c}\right) \mu
$$

provided $x_{P S}<x_{T}$.

First consider the case $\mu=0$ which corresponds to the situation described in section IV. Then on the sliding surface $\dot{x}=(a-b c) x$ and $x_{T}=0$ (the degenerate stationary point, see Figure 1a,b) . Hence if $a-b c>0$ solutions on the sliding manifold with $\mu=0$ move left (as $x<0$ on the sliding surface) whilst if $a-b c<0$ they move to the right, justifying the first case of the previous section.

To describe the dynamics as $\mu$ passes though zero it is necessary to understand the one-dimensional dynamics on the sliding manifold as a function of $\mu$ and the parameters. This depends crucially on the relative positions of $x_{P S}$ and $x_{T}$, and sometimes also with the intersection of the eigenvectors of the stable node with $y=0$ in $\mu>0$. In this case, the eigenvectors (diagonals through $(0, \mu)$ ) intersect $y=0$ at $x=-\mu$ and $x=\mu$. Moreover, at $x_{T}$ the dynamics on the sliding surface is

$$
\dot{x}=\frac{1}{D}\left[(a-b c) \frac{a}{b}+(a c-b)\right] \mu=\frac{1}{b D}\left(a^{2}-b^{2}\right) \mu
$$

and so at $x=x_{T}$, the tangency on the sliding surface,

$$
\dot{x}>0 \quad \text { if } \mu>0 ; \quad \dot{x}<0 \quad \text { if } \mu>0 \quad\left(\text { near } x_{T}\right) .
$$

Now the one-dimensional dynamics on the sliding surface can be assembled using this information near $x_{T}$ and then reversing the direction of flow as $x$ passes through a point where the sliding vector field vanishes, i.e. at a standard pseudo-equilibria. The only candidate for such a reversal is $x_{P S}$. To simplify the conditions let

$$
u=\frac{b-a c}{a-b c}
$$

so $x_{P S}=u \mu$ and $x_{T}=s \mu$ using (35), so the relative positions of each are determined by inequalities between $s$ and $u$ and also \pm 1 if $\mu>0$ and the eigenvectors of the stable node are important. Since $a-b<a+b<0$ almost all solutions tend to the node along the eigenvalue with slope +1 . The other eigenvector, which acts as a separatrix, intersects $y=0$ at $x=\mu$ if $\mu>0$. Solutions starting on $y=0$ to the right of this point tend to the stationary point on the right hand (upper) branch of the stable manifold and those with $x<\mu$ tend to the stationary point tangential to the left hand (lower) branch of the eigenvector.

The sliding manifold is $x<x_{T}$ and by assumption $s<-1$. Note that $b D>0$ implies that near $(x, \mu)=$ $(0,0)$ the sign of $\dot{x}$ on the sliding manifold is the sign of $\left(a^{2}-b^{2}\right) \mu$. The relative positions of the points $x_{T}=s \mu$ and $x_{P S}=u \mu$ depend on the sign of $\mu$.

Suppose that $\mu<0$. Then $x_{T}>0$ and $a^{2}>b^{2}$ so the direction of the sliding flow at $x_{T}$ is negative as shown in Figure 2a. The point $x_{P S}$ does not exist if $u \mu>s \mu$, i.e. if $u<s$ whilst it exists and is a pseudo-stable node if $u>s$ (as the sign of $\dot{x}$ on the sliding surface changes sign at $\left.x_{P S}\right)$.

Suppose that $\mu>0$. Then $x_{T}<0$ and $\dot{x}$ on the sliding manifold is positive at $x_{T}$. Thus if $u<s$ then $x_{P S}$ exists and is unstable in the sliding manifold.

Putting this together with the behaviour in $y>0$ shows that if $u>s$ there is a stable pseudo-node on the sliding surface for $\mu<0$ and a stable node in $\mu>0$ : stability is not changed. On the other hand if $u<s$ then if $\mu<0$ there are no stationary points locally and if $\mu>0$ the stable node in $y>0$ coexists with a pseudo-saddle on the sliding surface as shown in Figure 2a. This is a generalized saddle-node bifurcation. Note that $u<s<-1$ implies that the pseudo-saddle is always to the left of the intersection of the eigendirection $(1,1)^{T}$ of the stable node, which intersects $y=0$ at $(-\mu, 0)$ and hence the geometry is completely defined as in the figure. This will not always be the case below.

\section{UNSTABLE NODE}

If the hyperbolic stationary point of $f^{+}$is an unstable node (UN) then the eigenvalues $a \pm b$ of the linear part of the symmetric matrix version of (1) satisfy

$$
0<b<a
$$


(a)
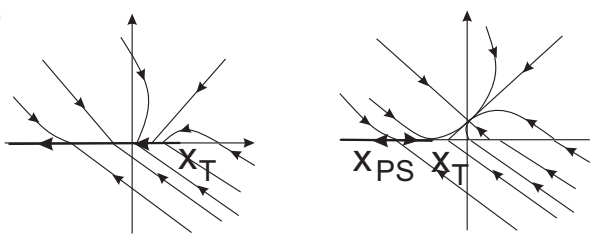

(b)
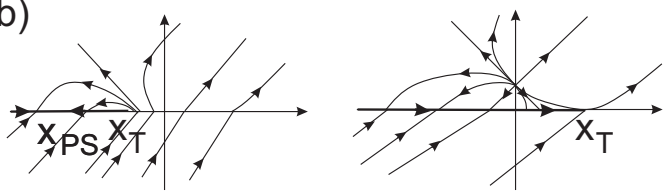

(c)
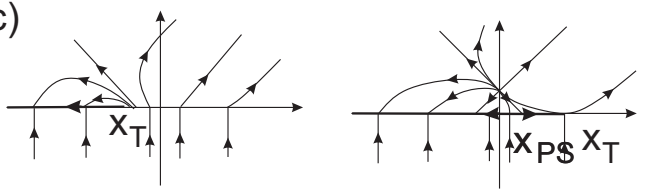

(d)

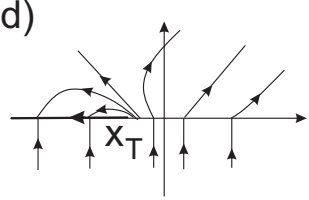

$\mu<0$

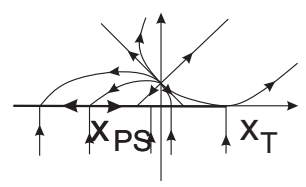

$\mu>0$

FIG. 2. Three of the four bifurcations of stable and unstable nodes, (c) and (d) are not topologically distinct, with $\mu<0$ on the left and $\mu>0$ on the right. (a) Generalized saddle-node bifurcation of pseudo-saddle and stable node. (b) Transition from stable pseudo-node to unstable node. (c) and (d) More generalized saddle-node bifurcations (cases with $|s|>1$ shown). Note that in (c) and (d) the stable manifold of the pseudo-saddle is tangential in negative time to opposite branches of the leading unstable eigenvector of the unstable node. In case (c) $u>-1$ and in case (d) $u<-1$, with $u$ as defined in (40).

so that the eigenvalues of $L, a \pm b$, are positive and distinct. The unstable node exists if $\mu>0$. From (32) and (33) the sliding surface is again given by $x<x_{T}=s \mu$ with $s=a / b>1$ from (35) and once again the pseudostationary point, $x_{P S}$, when it exists, is given by $x_{P S}=$ $u \mu$ with $u$ given by (40). As before, the sign of the sliding flow near $x_{T}$ equals the sign of $\left(a^{2}-b^{2}\right) \mu$ and if $\mu=0$ the flow on the sliding surface is $\dot{x}=(a-b c) x$ leading to the same direction of flow as in the stable node for this degenerate case, confirming the remarks for the unstable node in section IV.

Thus if $\mu<0$ and $u<s$ then there are no pseudostationary points on the sliding manifold locally and solutions move away from $x_{T}$ in the negative $x$-direction. If $\mu<0$ and $u>s$ then the pseudo-stationary point exists and is stable.

If $\mu>0$ and $u>s$ then there are no pseudo-stationary points and the unstable node exists in $y>0$. Thus if $u>$ $s$ there is a rather curious bifurcation with a transition from a stable pseudo-stationary point in $\mu<0$ to an unstable node in $\mu>0$. This is illustrated in Figure $2 \mathrm{~b}$.

If $\mu>0$ and $u<s$ then the pseudo-stationary point exists and is a saddle, unstable in the direction of the sliding surface (by consistency with the sign of $\left(a^{2}-b^{2}\right) \mu>0$ near $x_{T}$ ). This therefore coexists with the unstable node in $y>0$ and two cases can be distinguished. If $u<-1$ then the $y>0$ branch of the stable manifold of the pseudo-saddle tends to the unstable node in reverse time tangential to the negative branch of the weakly unstable direction, $(1,-1)^{T}$; whilst if $-1<u<s$ it tends to the unstable node tangential to the positive branch. This is illustrated in Figure 2c,d respectively. Topologically there is not a distinction between these two cases, so they are not divided in Hogan et $a l^{7}$, but the difference is worth observing nonetheless since the difference can have significance in applications. The condition $u>s$ (the strange bifurcation of Figure $2 \mathrm{~b}$ ) is equivalent to

$$
a-b c<0 \quad \text { or } \quad c>\frac{a}{b}
$$

whilst the more standard saddle-node bifurcation occurs if $a-b c>0$.

\section{SADDLE}

If the eigenvalues of the symmetric matrix $L_{0}$ of (2) have opposite signs then

$$
0<|a|<b .
$$

All the calculations of the previous two sections hold good here although the interpretation changes, and there are two cases, $a>0$ and $a<0$, which will be treated separately.

\section{A. Case SA: $a>0$.}

Unlike the previous sections $a^{2}-b^{2}<0$ and so the direction of the flow on the sliding manifold near $x_{T}$ is reversed: if $\mu<0$ it is positive and if $\mu<0$ it is negative. In this case $0<s<1, x_{T}=s \mu$.

If $\mu<0$ then $-|\mu|<x_{T}<0$ and the flow on the sliding surface is positive near $x_{T}$. There is no pseudo-stationary point if $u<s$ (recall the definitions of $s$ and $u$ in (35) and (40), whilst if $u>s$ then there is a pseudo-saddle, unstable in the sliding direction.

If $\mu>0$ then $0<x_{T}<\mu$ and the flow on the sliding surface is negative near $x_{T}$. If $u>s$ then there is no pseudo-stationary point and the saddle in $y>0$ exists, so in this case there is no bifurcation; a pseudo-saddle in $\mu<0$ becomes a standard saddle if $\mu>0$.

If $u<s$, so there is no pseudo-stationary point if $\mu<0$, then $\mu>0$ implies the existence of a stable pseudo-node, 

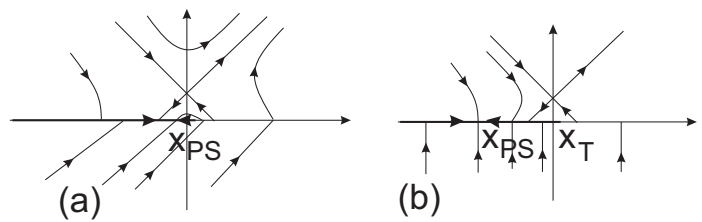

FIG. 3. Two of the three bifurcations of saddles showing $\mu>0$ only. (a) Generalized saddle-node bifurcation with $-1<u<$ $s$; the pseudo-node is approached in $y>0$ by a trajectory that comes from $y<0$. (b) Generalized saddle-node bifurcation with $u<-1$. Here the pseudo-node is approached from $y>0$ by a trajectory that stays in $y>0$.

so there is a non-standard saddle-node bifurcation with the creation of a saddle and stable pseudo-node as $\mu$ passes through zero. This bifurcation has two subcases as illustrated in Figure 3. If $-1<u<s$ then (since $s<1$ ) the pseudo-node $x_{P S}$ lies between intersections of the stable and unstable manifolds of the saddle with $y=0$, and hence the solution that tends to $x_{P S}$ from $y>0$ actually comes from the region $y<0$, whilst if $u<-1$ then $x_{P S}$ lies to the left of the unstable manifold of the saddle and comes from $y>0$. This reflects a genuine topological difference between the two cases (unlike the distinction made earlier for the unstable node). The condition $-1<u<s$ is $\frac{a}{b}<c<1$, and $u<-1$ implies $c>1$.

\section{B. Case SB: $a<0$.}

If $a<0$ then $-1<s<0$ and the cases are similar.

If $\mu<0$ then $0<x_{T}<|\mu|$ and the direction of the flow on the sliding manifold near $x_{T}$ is positive. As in Case SA, there is no pseudo-stationary point if $u<s$ and if $u>s$ then there is a pseudo-saddle, unstable in the sliding direction. If $\mu>0$ then the direction of the flow on the sliding manifold near $x_{T}$ is negative. As in Case SA there is no pseudo-stationary point if $u>s$ and a stable pseudo-node if $u<s$. In the former case there is a transition from a pseudo-saddle to a standard saddle as $\mu$ passes through zero and the latter case is a generalized saddle-node bifurcation. The two cases $-1<u<s$ and $u<-1$ are distinguished in the same way as in the case SA.

\section{STABLE FOCUS}

For the stable focus and the unstable focus of the next section the linear part of the normal form is antisymmetric $L_{1}$ of (2) with $b>0$ which implies that the rotation is counterclockwise and so the switching surface is in $x<x_{T}=s \mu, s=a / b$ (as before, see (35)). In this case the flow on the sliding manifold is

$$
\dot{x}=\frac{1}{D}[(a-b c) x+(b+a c) \mu]
$$

(a)

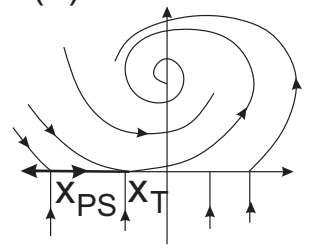

(c)

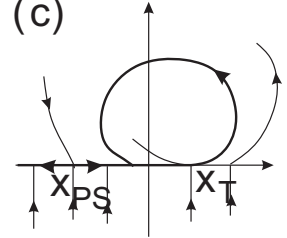

(b)

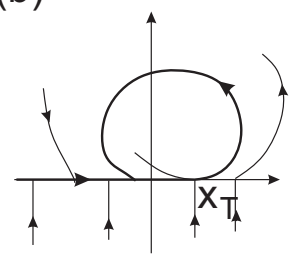

(d)

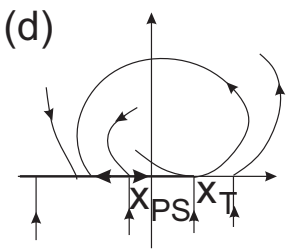

FIG. 4. Four of the five bifurcations of stable and unstable foci showing $\mu>0$ only. (a) Generalized saddle-node bifurcation of pseudo-saddle and stable focus. (b) Stable sliding orbit with unstable focus. (c) Stable sliding orbit with pseudosaddle and unstable focus. (d) Unstable focus and pseudosaddle.

with $D$ given by (37) and so $x_{P S}=v \mu$ with

$$
v=\frac{a c+b}{b c-a}
$$

provided $x_{P S}<x_{T}$. At $x_{T}(44)$ is

$$
\dot{x}=\frac{1}{b D}\left(a^{2}+b^{2}\right) \mu
$$

and so, since $D>0$ locally, the direction of the sliding flow near $x_{T}$ is determined by the sign of $\mu$ (positive if $\mu>0$ and negative if $\mu<0$ ).

Thus for the stable focus

$$
a<0, \quad b>0, \quad(s<0)
$$

and so if $\mu<0$ and $v<s$ then $x_{T}>0$ and there is no pseudo-stationary point and sliding solutions move left, whilst if $\mu<0$ and $v>s$ then there is a stable pseudonode.

If $\mu>0$ then $x_{T}<0$ and a pseudo-saddle exists if $v<s$, whilst no pseudo-stationary points exist if $v>s$ and all solutions on the sliding manifold move up to $x_{T}$ and from there to the stable focus in $y>0$.

Thus if $v>s$ a stable pseudo-node becomes a stable focus as the parameter varies through zero. If $v<s$ then there is a generalized saddle-node bifurcation (or more accurately, a saddle-focus!) as shown in Figure 4a, in which a pseudo-saddle and stable focus are created as the bifurcation parameter is varied through zero. Note that this latter condition, $v<s$, is equivalent to $a-b c>0$ or $c<\frac{a}{b}$. 


\section{UNSTABLE FOCUS}

This case is rather more interesting. The linear part of the normal form is $L_{1}$ of (2) with parameters

$$
a>0, \quad b>0, \quad(s>0) .
$$

The definitions of $x_{T}, x_{P S}, s$ and $v$ are as in section VIII and the direction of the sliding flow near $x_{T}$ is again determined by (46).

If $\mu<0$ then there are two cases. If $v<s$ then there are no pseudo-stationary points on the sliding manifold locally and solutions slide to the left. If $v>s$ then there is a stable pseudo-node on the sliding surface.

If $\mu>0$ then again there are two possible cases for the existence of pseudo-stationary points. If $v>s$ then there are no pseudo-stationary points and solutions slide to the right, whilst if $v<s$ there is a pseudo-saddle at $x_{P S}=v \mu$ and solutions to the right of this point slide towards $x_{T}$.

In previous cases this, together with the linear flow in $y>0$ was enough to determine the behaviour of solutions. In this case solutions that reach the tangency point $x_{T}$ along the sliding manifold may return to the sliding manifold and thence slide to $x_{T}$ forming a sliding periodic orbit. This could not happen in previous cases. If $v>s$ and $\mu>0$ then the solution in $y>0$ through $\left(x_{T}, 0\right)$ will return to $y=0$ with $x<x_{T}$ after a time less than $2 \pi / b$ and then slide back to $x_{T}$ creating the sliding orbit as shown in Figure 4b.

If $v<s$ then the situation is a little more complicated, see Figure 4c,d. As before, the solution through $\left(x_{T}, 0\right)$ will return to $y=0$ for the first time at some point $x=h \mu$, but this time if $h<v$ then the solution is on the 'other' side of the pseudo-saddle and will not return to $x_{T}$, whilst if $h>v$ then there will be a sliding periodic orbit. At the boundary between these two cases, $h=v$, there is a homoclinic orbit from $x_{P S}$ to itself that includes a sliding component. This condition can be expressed implicitly, and (for the sake of completeness) we give the rather uninformative expressions.

By simple geometry (using the sine rule) an expression for the angle $\psi$ subtended at the unstable focus with the points $x_{P S}$ and $x_{T}$ can be obtained, and the time $\tau_{h}$ to pass through $2 \pi-\psi$ is obtained by dividing by $b$ giving

$$
\tau_{h}=\frac{1}{b}\left(2 \pi-\sin ^{-1}\left(\frac{s-v}{\sqrt{v^{2}+1} \sqrt{s^{2}+1}}\right)\right) .
$$

Thus the special solution exists if the solution in $y>0$, $y(t)$ satisfies $y\left(\tau_{h}\right)=0$ and $y(t)>0,0<t<\tau_{h}$. The first of these conditions is (after cancelling a factor of $\mu$ )

$$
e^{a \tau_{h}}\left(\cos \left(b \tau_{h}\right)-\frac{a}{b} \sin \left(b \tau_{h}\right)\right)=1
$$

giving the implicit conditions for the existence of the critical parameter values.

\section{SUMMARY}

In this section the results on codimension one border equilibrium bifurcations of (1) with either $L_{0}$ or $L_{1}$ defined by (2) with a description of the dynamics as $\mu$ passes through zero. The connection to Filippov's description ${ }^{5}$ is given along with the relevant case from Hogan et $a l^{7}$ in the order they appear in their Table 1. Following Hogan et $a l^{7}$ we use the symbols $p, n, f$, and $s$ to indicate pseudo, node, focus and saddle and add spo for sliding periodic orbit and subscripts $s$ or $u$ to indicate stability (stable or unstable). Bifurcation transitions are indicated by a double arrow.

\section{A. Stable Node (Section V)}

In this case the linear part is $L_{0}$ with $a<0,0<b<|a|$.

- If $c>\frac{a}{b}$ (i.e. $u>s$ ) then a stable pseudo-node exists in $\mu<0$ and a stable node in $\mu>0$. This is described in Figure 101 of Filippov ${ }^{5}$ and is the first case of Hogan et $a l^{7}$ with transition $p n_{s} \leftrightarrow n_{s}$.

- If $c<\frac{a}{b}$ then there are no stationary points or pseudostationary points if $\mu<0$ and a pseudo-saddle coexists with a stable node if $\mu>0$. This is a generalized saddle-node bifurcation illustrated in Figure 2a corresponding to an unfolding of Figure 93 of Filippov ${ }^{5}$ and is Hogan et al's third case ${ }^{7}$ (one of the two 'missing' cases) with transition $\emptyset \leftrightarrow p s+n_{s}$.

\section{B. Unstable Node (Section VI)}

In this case the linear part is $L_{0}$ with $0<b<a$.

- If $c>\frac{a}{b}$ then a stable pseudo-node exists if $\mu<0$ and an unstable node if $\mu>0$, a bifurcation illustrated in Figure 2b. This is the interesting 'missing' case of an unfolding of Filippov's Figure $92^{5}$ and is case four of Hogan $e t a l^{7}$ with transition $p n_{s} \leftrightarrow n_{u}$.

- If $c<\frac{a}{b}$ then there are no stationary points or pseudostationary points locally if $\mu<0$ and a pseudo-saddle and unstable node if $\mu>0$. This is a generalized saddle-node bifurcation corresponding to an unfolding of Figure 94 of Filippov ${ }^{5}$ and is case 1 of Hogan et $a l^{7}$ with the transition $\emptyset \leftrightarrow p s+n_{u}$. If $c>1$ (i.e. $u<-1$ )then the branch of the stable manifold of the pseudo-saddle approaches the node in reverse time tangential to the negative $(x<0)$ branch of the weakly unstable eigenvector of the node, whilst if $c<1$ the tangential approach is to the positive branch. See Figures $2 \mathrm{c}, \mathrm{d}$. Note that this is not a topological distinction, but it may be important in examples. 


\section{Saddle (Section VII)}

In this case the linear part is $L_{0}$ with $0<|a|<b$.

- If $c<\frac{a}{b}$ (i.e. $u>s$ ) then there is a pseudo-saddle if $\mu<0$ and a saddle if $\mu>0$. This corresponds to an unfolding of Filippov's Figure $90^{5}$ and case 7 of Hogan et $a l^{7}$ with transition $p s \leftrightarrow s$.

- If $c>\frac{a}{b}$ and $u<-1$, i.e. if $\frac{a}{b}<c<1$, then there are no stationary points or pseudo-stationary points if $\mu<$ 0 and a stable pseudo-node coexists with a saddle if $\mu>0$ so there is a generalized saddle-node bifurcation and the trajectory that approaches the pseudo-node from $y>0$ lies in $y>0$ for all negative time. This is Filippov's Figure $100^{5}$ and case 5 of Hogan et $a l^{7}$ with transition $\emptyset \leftrightarrow p s+s$.

- If $c>\frac{a}{b}$ and $u>-1$, i.e. if $c<1$, then there are no stationary points or pseudo-stationary points if $\mu<0$ and a stable pseudo-node coexists with a saddle if $\mu>$ 0 so there is a generalized saddle-node bifurcation and the trajectory that approaches the pseudo-node from $y>0$ lies in $y<0$ for all sufficiently large and negative times. This is an unfolding of Filippov's Figure $89^{5}$ and case 6 of Hogan et $a l^{7}$ with transition $\emptyset \leftrightarrow p s+s$.

\section{Stable Focus (Section VIII)}

In this case the linear part is $L_{1}$ with $a<0<b$.

- If $c>\frac{a}{b}$ (i.e. $v>s$ ) then a stable pseudo-node exists if $\mu<0$ and if $\mu>0$ there is a stable focus. The transition is thus $p n_{s} \leftrightarrow f_{s}$ and it is an unfolding of Filippov's Figure $95^{5}$ and case 11 of Hogan et al .

- If $c<\frac{a}{b}$ then there are no stationary points or pseudostationary points locally if $\mu<0$ whilst a pseudosaddle coexists with the stable focus if $\mu>0$, so there is a generalized saddle-node bifurcation. The flow if $\mu>0$ is sketched in Figure 4a. The transition is therefore $\emptyset \leftrightarrow p s+f_{s}$ which is an unfolding of Filippov's Figure 96 and case 12 of Hogan et $\mathrm{al}^{7}$.

\section{E. Unstable Focus (Section IX)}

In this case the linear part is $L_{1}$ with $0<a, b$.

- If $c>\frac{a}{b}$ (i.e. $v>s$ ) then a stable pseudo-node exists if $\mu<0$ and if $\mu>0$ there is a stable sliding periodic orbit enclosing an unstable focus as shown in Figure $4 \mathrm{~b}$. The transition is thus $p n_{s} \leftrightarrow s p o_{s}+f_{u}$ which is an unfolding of Filippov's Figure $95^{5}$ and case 10 of Hogan et $a l^{7}$.

- If $c<\frac{a}{b}$ and $h<v$ (see Section IX) then there are no stationary points or pseudo-stationary points locally if $\mu<0$ whilst a pseudo-saddle coexists with the unstable focus if $\mu>0$ (Figure $4 \mathrm{~d}$ ). The transition is $\emptyset \leftrightarrow p s+f_{u}$ which is Filippov's Figure $102^{5}$ and case 9 of Hogan et $a l^{7}$.

- If $c<\frac{a}{b}$ and $h<v$ (see Section IX) then there are no stationary points or pseudo-stationary points locally if $\mu<0$ whilst a pseudo-saddle coexists with the unstable focus and a stable sliding periosic orbit if $\mu>0$ as shown in Figure 4c. The transition is $\emptyset \leftrightarrow p s+s p o_{s}+f_{u}$ which is Filippov's Figure $98^{5}$ and case 8 of Hogan et $a l^{7}$.

The boundary between these cases is given implicitly by (49), (50).

We have thus isolated twelve topologically distinct cases (one of which, the generalized saddle-node bifurcation of the unstable node) was divided further into two cases depending on which branch of the weak unstable manifold of the unstable node the stable manifold of the pseudo-saddle tends to in reverse time. These twelve cases are precisely the cases described by Filippov ${ }^{5}$ and Hogan et $a l^{7}$. The creation of pairs of stationary (or pseudo-stationary) points in generalized saddle-node bifurcations leads to points that are separated by a distance of order $|\mu|$, unlike the characteristic $\sqrt{|\mu|}$ separation of smooth saddle-node bifurcations. Similarly, the growth of the amplitude of sliding periodic orbits is again proportional to $|\mu|$ rather than the $\sqrt{|\mu|}$ of standard Hopf bifurcations.

\section{CODIMENSION TWO BIFURCATIONS}

The boundaries between any two of the codimension one bifurcation regions described above provides a natural way of discussing some of the codimension two bifurcations of planar Filippov systems. For example if the linear part of the normal form is given by the antisymmetric $L_{1}$ then the boundary between the stable and unstable focus cases is the codimension two Boundary Hopf bifurcation described by Dercole et $a l^{4}$.

In this section a codimension two bifurcation will be considered associated with the condition $c=\frac{a}{b}$ which is on the boundary between many of the cases described in previous section. This is not an obvious choice without having seen the analysis of previous sections. Consider the two cases of Figure 2b,d: in the former case there is a transition from a codimension one bifurcation in which a stable pseudo-node becomes an unstable focus and a generalized saddle-node bifurcation. To make matters more complicated the far-field flow on the sliding surface must change direction, which suggests that higher order terms become significant.

Both bifurcations are described in section VI with $0<b<a$ in $L_{0}$ of (2). The first has $u>s$ with $s$ and $u$ defined by (35) and (40), which implies that $a<b c$ and $a^{2}<b^{2}$ (the latter of which is automatically satisfied). In particular $c$ must be positive. The second has $u<s$ 

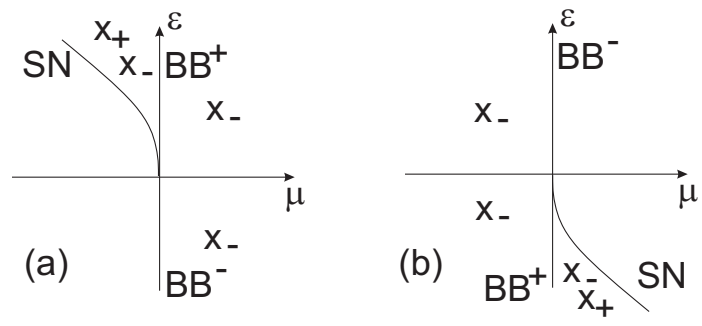

FIG. 5. The codimension two parameter space; the existence of pseudo-equilibria is indicated using the symbols $x_{ \pm}$. The loci of the one-dimensional boundary bifurcations $B B^{ \pm}$are the half-axes with $\mu=0 . S N$ denotes the locus of a pseudosaddle-node bifurcation. (a) $R<0$ : $x_{-}$is a pseudo-saddle and $x_{+}$a stable pseudo-node. (b) $R>0: x_{-}$is a stable pseudo-node and $x_{+}$a pseudo-saddle.

(with $u>-1$ ) and has a pseudo-saddle and the unstable focus coexisting in $\mu>0$. Thus the codimension two situation is $u=s$, i.e. $c=\frac{a}{b}, \mu=0$, with $0<b<a$. As noted earlier, section $\mathrm{X}$ shows that this is an important codimension two situation in many bifurcations, and the local bifurcation diagram of Figure 5 derived in this section will be relevant for all these cases, but with a different interpretation of the upper and lower flows. The condition implies that the linear terms in $x$ of the sliding flow vanishes and so there is a classic bifurcation in the sliding manifold itself.

Setting $c=\frac{a}{b}+\epsilon$, the description of the bifurcations in section VI together with equation (42) shows that if $\epsilon>0$ the bifurcation as $\mu$ passes through zero has the interesting transition from a stable pseudo-node to an unstable node, whilst if $\epsilon<0$ then there is a more standard saddle-node bifurcation. To analyze the dynamics near the codimension two point $(\mu, \epsilon)=(0,0)$ first note that since both the individual flows $f^{ \pm}$are hyperbolic, the behaviour off the sliding surface does not change significantly. On the sliding surface $x<s \mu(s=a / b)$ the flow (36) becomes

$$
\dot{x}=\frac{1}{b D}\left(\left(a^{2}-b^{2}\right) \mu-b^{2} \epsilon x+R x^{2}+\ldots\right)
$$

where the coefficient $R$ depends on the details of the particular flow and is typically non-zero. The local bifurcation structure of this equation is simply a matter of calculation with quadratics and some irritating inequalities, and the results are sketched in Figure 5, where $B B^{ \pm}$ refers to a boundary bifurcation in which $x_{ \pm}$coincides with the tangency point $x_{T}=s \mu$. This creates or destroys a pseudo-equilibrium. The full two-dimensional flows can be obtained by adding the hyperbolic flows of the systems $f^{ \pm}$to this picture. The bifurcation sequence is different depending on the sign of $R$.

Suppose $R<0$ as shown in Figure 5a. If $\epsilon>0$ then as $\mu$ increases from a negative value, initially there are no pseudo-stationary points and orbits slide left, then a saddle-node bifurcation creates a pair $x_{ \pm}$of pseudostationary points: $x_{-}$is a saddle, unstable in the slid- ing manifold, and $x_{+}$is stable. As $\mu$ increases further the stable pseudo-stationary point $x_{+}$is destroyed in a boundary bifurcation $B B^{+}$at $\mu=0$ and the unstable node in $y>0$ is created, so if $\mu>0$ the unstable node coexists with the pseudo-saddle, whose role is essentially to ensure that the stability of the bifurcating solutions is compatible with the constraints due to the sign of $R$.

If $\epsilon<0$ then initially there are no pseudo-stationary points, and as $\mu$ passes through zero a pseudo-saddle, $x_{-}$ is created by boundary bifurcation and the unstable node in $y>0$ is also created: effectively this is a saddle-node bifurcation.

Now suppose that $R>0$ (Figure 5b). If $\epsilon>0$ then for $\mu<0$ there is a stable pseudo-node $x_{-}$, and this is destroyed by a boundary bifurcation at $\mu=0$ which also creates the unstable node in $y>0$. If $\epsilon<0$ then as $\mu$ is increased, the stable pseudo-node $x_{-}$exists and as $\mu$ passes through zero a pseudo-saddle is created by a boundary bifurcation that also creates the unstable node in $y>0$. This is the standard local picture. As $\mu$ increases further the two pseudo-stationary orbits are destroyed by a saddle-node bifurcation on the sliding manifold leaving only the unstable node in $y>0$.

Other cases can be considered similarly and those that involve the criterion $c=\frac{a}{b}$ all have the same local picture on the sliding surface, but with mild re-interpretations for the full planar system.

\section{CONCLUSION}

By deriving a lowest order normal form for boundary equilibrium bifurcations it is possible to understand the list provided by Filippov ${ }^{5}$ and ensure that all cases have been considered systematically. The analysis also draws attention to codimension two points that separate the different codimension one bifurcations described. This provides a particularly simple framework within which to describe the bifurcations of planar systems and it may be possible to extend these ideas to higher dimensions.

Acknowledgements: This paper was written as a result of a series of interesting conversations with John Hogan. I am grateful to John Hogan and Mike Jeffrey for comments. This work was partly funded by the EPSRC Platform Grant (MAPLE) EP/I01912X/1.

\footnotetext{
${ }^{1}$ M. di Bernardo, C. Budd, A.R. Champneys and P. Kowalczyk, P. (2008) Piecewise-Smooth Dynamical Systems, Springer, London. ${ }^{2}$ M. di Bernardo, D.J. Pagano and E. Ponce (2008) Nonhyperbolic boundary equilibrium bifurcations in planar Filippov systems: a case study approach, Int. J. Bifurcation Chaos 18 1377-1392.

${ }^{3}$ T. de Carvalho and D.J. Tonon (2014) Normal forms for codimension one planar piecewise smooth vector fields, Int. J. Bifurcation Chaos 24 1450090, DOI:10.1142/S0218127414500904.

${ }^{4}$ F. Dercole, F. Della Rossa, A. Colombo and Y.A. Kuznetsov (2011) Two Degenerate Boundary Equilibrium Bifurcations in Planar Filippov Systems, SIADS 10 1525-1553.

${ }^{5}$ A.F. Filippov (1988) Differential Equations with discontinuous right hand sides, Kluwer, Dordrecht.
} 
${ }^{6}$ M. Guardia, T.M. Seara, and M.A. Teixeira (2011) Generic bifurcations of low codimension of planar Filippov Systems, J. Diff. Equ. 250 1967-2023.

${ }^{7}$ S.J. Hogan, M.E. Homer, M.R. Jeffrey, R. Szalai (2015) Piecewise smooth dynamical systems: the case of the missing boundary equilibrium bifurcations, preprint, University of Bristol.

${ }^{8}$ Y.A. Kuznetsov, S. Rinaldi and A. Gragnani (2003) One-
Parameter Bifurcations in Planar Filippov Systems, Int. J. Bifurcation Chaos 13 2157-2188.

${ }^{9}$ H.E. Nusse and J.A. Yorke (1992) Border-collision bifurcation including 'period two to period three' for piecewise smooth systems, Physica D 57 39-57. 